2021-1

\title{
Optimization of Turbine Tilt in a Wind Farm
}

James Cutler

Brigham Young University, cutlerjames36@gmail.com

Andrew P.J. Stanley

Brigham Young University, PJ.Stanley@nrel.gov

Jared J. Thomas

Brigham Young University, jaredthomas68@gmail.com

Andrew Ning

Brigham Young University, aning@byu.edu

Follow this and additional works at: https://scholarsarchive.byu.edu/facpub

Part of the Mechanical Engineering Commons

\section{Original Publication Citation}

Cutler, J., Stanley, A. P. J., Thomas, J. J., and Ning, A., "Optimization of Turbine Tilt in a Wind Farm," AIAA SciTech Forum, virtual, Jan. 2021. doi:10.2514/6.2021-1180

\section{BYU ScholarsArchive Citation}

Cutler, James; Stanley, Andrew P.J.; Thomas, Jared J.; and Ning, Andrew, "Optimization of Turbine Tilt in a Wind Farm" (2021). Faculty Publications. 4550.

https://scholarsarchive.byu.edu/facpub/4550

This Conference Paper is brought to you for free and open access by BYU ScholarsArchive. It has been accepted for inclusion in Faculty Publications by an authorized administrator of BYU ScholarsArchive. For more information, please contact ellen_amatangelo@byu.edu. 


\title{
Optimization of Turbine Tilt in a Wind Farm
}

\author{
James Cutler*, Andrew P.J. Stanley ${ }^{\dagger}$, Jared J. Thomas ${ }^{\ddagger}$, and Andrew Ning ${ }^{\S}$ \\ Brigham Young University, Department of Mechanical Engineering, Provo, UT, 84602
}

\begin{abstract}
Wind farm power production is significantly affected by upstream turbines creating wakes of slower wind speeds that overlap the rotor swept areas of downstream turbines. By optimizing the tilt angle of the turbines in a farm, wakes may be deflected away from downstream turbines, increasing the overall energy production. In this study, we optimized the tilt angle of turbines in a wind farm to maximize energy production. We used an analytic wake model modified for gradient-based optimization to consider wake deflection from tilt. We considered optimizing the tilt angle of each turbine assuming that it remained fixed for the lifetime of the farm. We also considered active tilt control. In a test case using the Princess Amalia 60-turbine wind farm, optimizing fixed tilt angles resulted in a $2.77 \%$ increase in the annual energy production(AEP) while using active tilt control resulted in an $13.64 \%$ increase in AEP.
\end{abstract}

\section{Introduction}

A $s$ the world continues to pursue renewable energy goals, it is increasingly important to improve the efficiency of wind farms. Wind farms lose between $15 \%$ and $20 \%$ of energy production for a typical wind farm throughout the year because of wake interference between turbines [1-4], and up to $40 \%$ for farms with closely spaced wind turbines [5]. One method that is currently used to reduce wake interference between turbines in a farm is through active control. Many studies have found that by yawing turbines, the wakes can be deflected and steered away from downstream turbines. Yawed turbines produce less power; however, the reduced waking of downstream turbines can result in a net gain [6-9]. Gebraad et al. observed that with optimal yaw angles the net AEP for a simple 2- by 3-wind turbine wind farm can increase by as much as $13 \%$ [10]. Gebraad et al. also observed that along with increasing annual energy production another benefit of yawing is a decrease in the loads experienced on the yawed turbines [6]. Conversely, due to the reduction in rotor swept area from yawing turbines the wake deflection may sometimes not be enough and there can be a net decrease in the energy production of the farm [3].

A similar method that has not been explored as thoroughly is wake deflection through turbine tilt. Just as yaw control can be used to deflect downstream wakes in the horizontal plane, tilt control can be used to deflect wakes up and down. The benefits of tilting turbines are not as easy to research, mainly because turbines do not yet have active tilt capabilities making it difficult to verify results in a real wind farm. However, there has been some research done exploring turbine tilt. An early study by Guntur et al. found that similar to yaw, tilt in a wind turbine can effectively deflect the wake. However, they suggest active tilt control appears to be rather impractical because the amount of tilt needed for significant gains in power production could run the blades into the tower [11].

More recent studies indicate that there may be significant benefits to wake deflection from turbine tilt. Annoni et al. analyzed possible increases in the energy production due to controlling tilt in a row of two of NREL's 5-MW reference turbines. Using the large-eddy simulation tool SOWFA (Simulator fOr Wind Farm Applications) [12], they found that when the turbines were placed 7 rotor diameters apart a positive tilt of about $25^{\circ}$ in the front turbine deflected the wake enough for an $8.3 \%$ net increase in energy production [13]. However, such results do not attempt to find the optimal angles of wind turbines in a wind farm because the simulation would be too computationally expensive.

This research will go beyond tilt control in a few turbines in a row with one wind direction, and explore tilt angle and active tilt control optimization for a full wind farm. By using an analytic wake model, we were able to quickly model an entire farm, and study the benefits of wake deflection from turbine tilt in a wind farm. We considered optimizing a fixed tilt angle for each turbine, assuming the same tilt angle for all wind directions. We also considered active control of turbine tilt. A tilt mechanism would be expensive and complicated to engineer, as well as increase turbine capital costs. However, this study is an early step in determining if the benefits of active tilt control are worth the difficulty of implementing it in real wind farms.

\footnotetext{
*Undergraduate Research Assistant

${ }^{\dagger} \mathrm{PhD}$ Candidate

$¥ \mathrm{PhD}$ Candidate

${ }^{\S}$ Associate Professor
} 


\section{Methods}

\section{A. Wake Model}

In this section we discuss the wake model used to predict wake interactions in order to calculate AEP. We used a modified version of the Bastankhah model (shown in Eq. 1), an analytic Gaussian wake model [14]. The original formulation of the Bastankhah model does not accurately model the near wake of a wind turbine, but it can accurately model the far wake. In this paper we only considered wind farms with turbines spaced relatively far apart (greater than 5 rotor diameters) such that only far wake interactions are relevant. In their paper, Bastankhah et al. state that their model can be used to predict wake deflection from turbine tilt in a similar manner to how they predict deflection from yaw. Thus, we modified their equations to consider turbine tilt in the wind speed deficit and wake deflection calculations (negative tilt is moving the blades away from the tower if the wind turbine is facing the wind) [14]. However, it is important to note that Bastankhah et al. stated their model does not accurately include the effect of the ground on the wake.

$$
\frac{\Delta \bar{u}}{\bar{u}_{\infty}}=\left(1-\sqrt{1-\frac{C_{T} \cos (\gamma)}{8\left(\sigma_{y} \sigma_{z} / d^{2}\right)}}\right) \exp \left[-0.5\left(\frac{y-y_{h}}{\sigma_{y}}\right)^{2}\right] \exp \left[-0.5\left(\frac{z-\delta}{\sigma_{z}}\right)^{2}\right]
$$

Therefore, we visually compared the velocity profiles of our modified Bastankhah model with the SOWFA simulations used by Annoni et al. in their assessment of turbine tilt [13]. Initially we noticed that for the case of tilting a turbine $-25^{\circ}$, shown in Fig. 11 the SOWFA simulation shows a more elliptical wake shape that is stretched out of the upper left corner of the rotor swept area whereas the Bastankhah wake model by default assumes no veer and therefore has a circular wake shape. Abkar et al. modified the Bastankhah wake model to include veer, represented by the $x \tan \left(\alpha_{i n}\right)$ term, for the incoming wind in the rotor swept area of a turbine [15](shown in Eq.2]and Eq. 33). By including veer in our wake model we were better able to replicate the wake shape for a $-25^{\circ}$ tilted turbine as seen in Annoni et. al's SOWFA simulation (see Fig. 1).

$$
\begin{gathered}
\alpha_{i n} \approx\left(\frac{\alpha_{t t}-\alpha_{b t}}{d}\right)\left(z-z_{h u b}\right) \\
\frac{\Delta \bar{u}}{\bar{u}_{\infty}}=\left(1-\sqrt{1-\frac{C_{T} \cos (\gamma)}{8\left(\sigma_{y} \sigma_{z} / d^{2}\right)}}\right) \exp \left[-0.5\left(\frac{y-y_{h}+x \tan \left(\alpha_{i n}\right)}{\sigma_{y}}\right)^{2}\right] \exp \left[-0.5\left(\frac{z-\delta}{\sigma_{z}}\right)^{2}\right]
\end{gathered}
$$

Equation 3 includes the thrust coefficient $\left(C_{T}\right)$, rotor diameter $(d)$, turbine tilt angle $(\gamma)$, the distance between the point of interest and the wake center in the cross-stream horizontal $\left(y-y_{h}+x \tan \left(\alpha_{i n}\right)\right)$ and vertical $(z-\delta)$ directions, the incoming wind angle $\left(\alpha_{i n}\right)$, and the wake widths in the cross-stream horizontal and vertical directions $\left(\sigma_{y}\right.$ and $\left.\sigma_{z}\right)$.

The wake widths, $\sigma_{y}$ and $\sigma_{z}$, are defined in Eqs. 4 and 5

$$
\begin{gathered}
\frac{\sigma_{y}}{d}=k_{y} \frac{x-x_{0}}{d}+\frac{1}{\sqrt{8}} \\
\frac{\sigma_{z}}{d}=k_{z} \frac{x-x_{0}}{d}+\frac{\cos (\gamma)}{\sqrt{8}}
\end{gathered}
$$

In these equations, $x$ is the downstream distance from the wind turbine creating the wake, $d$ is the rotor diameter of the turbine, $x_{0}$ is the length of the wake potential core, and $k_{y}$ and $k_{z}$ are constants with the values of .025 and .0175 respectively [16]. The smaller value associated with $k_{z}$ accounts for some of the effect of the ground on the wake expansion. The length of the potential core is defined by Eq. 6 where $\alpha=2.32, \beta=0.154$, and $I=0.06$.

$$
\frac{x_{0}}{d}=\frac{\cos (\gamma)\left(1+\sqrt{1-C_{T}}\right)}{\sqrt{2}\left(\alpha I+\beta\left(1-\sqrt{1-C_{T}}\right)\right.}
$$

Interacting wakes are defined with linear superposition, as described by Niayifar et al. [17]. 

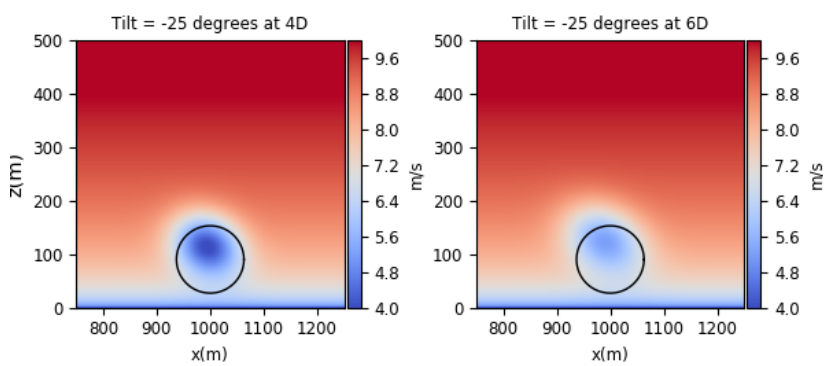

(a) Modified Bastankhah Model

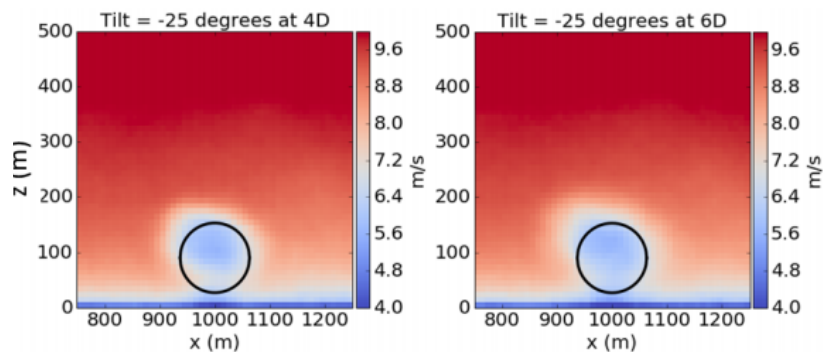

(b) SOWFA

Figure 1 Stream-wise component of velocity of cross sections of a -25 degree tilted wind turbine at 4D and 6D downstream from the (a) modified Bastankhah model and (b) NREL's SOWFA simulations [13].

To further validate the wake model we compared the velocity profiles for a turbine tilted 25 degrees and -25 degrees (see Fig. 1 and Fig. 2). Generally, the wake shapes and velocity deficits match. The largest differences occur when the turbine is tilted $25^{\circ}$. At 6 rotor diameters downstream, the SOWFA simulation depicts a kidney bean shape where higher velocity deficits appear on the edges of the rotor swept area. Our wake model doesn't account for this shape which leads to our wake model under predicting power gains from using tilt. We noticed that SOWFA showed the wake deflecting slightly more with positive tilt than negative tilt (see Fig. 3.
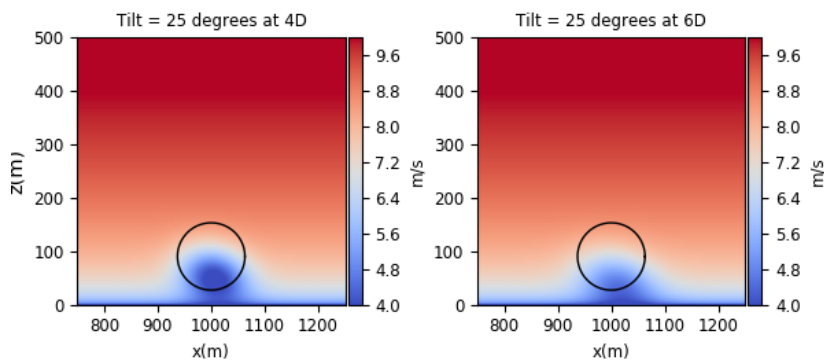

(a) Modified Bastankhah Model

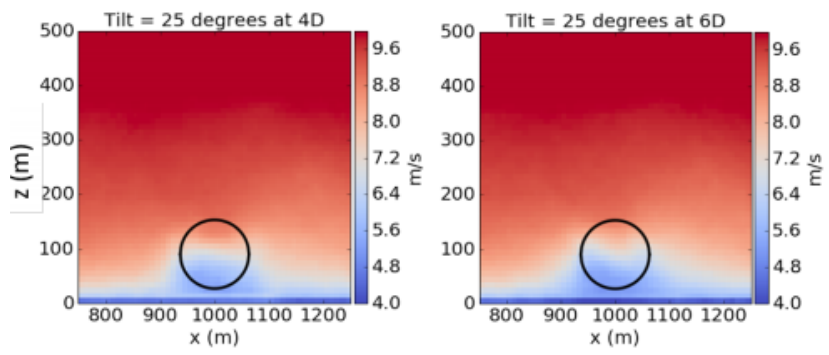

(b) SOWFA

Figure 2 Stream-wise component of velocity of cross sections of a 25 degree tilted wind turbine at 4D and 6D downstream from the (a) modified Bastankhah model and (b) NREL's SOWFA simulations [13]. 
To account for the added deflection with positive tilt we noticed that, for whatever intended tilt angle we were modeling, if we added around $5^{\circ}$ of tilt to the wake model we were better able to match SOWFA. For example, if the intended tilt angle was $25^{\circ}$ then the model would use $30^{\circ}$ to define the deflection and $25^{\circ}$ to calculate the power. In Fig. 3 this offset can be seen in the comparison of stream-wise velocity profiles for the modified Bastankhah model and Annoni et al.'s SOWFA runs. As mentioned earlier, the Bastankhah wake model is accurate after about 4 rotor diameters downstream. Therefore, we tuned our modified model to match SOWFA's stream-wise velocity profiles around 6 to 7 rotor diameters downstream. At 6 rotor diameters downstream the stream-wise velocity profile has a lower maximum wind speed with a $-25^{\circ}$ tilt angle than a $25^{\circ}$ tilt angle. This pattern matches SOWFA's stream-wise velocity profiles except that the modified Bastankhah model over predicts the wind speeds in the bottom portion of the wake from a $-25^{\circ}$ tilt. This leads to an over prediction of power gains for wind turbine arrays with negative tilt. However, since positive tilt results in significantly higher power gains than negative tilt, while also under predicting power gains compared to SOWFA, we predict the optimization will be able to sufficiently estimate the power gains of turbine tilt in a wind farm.

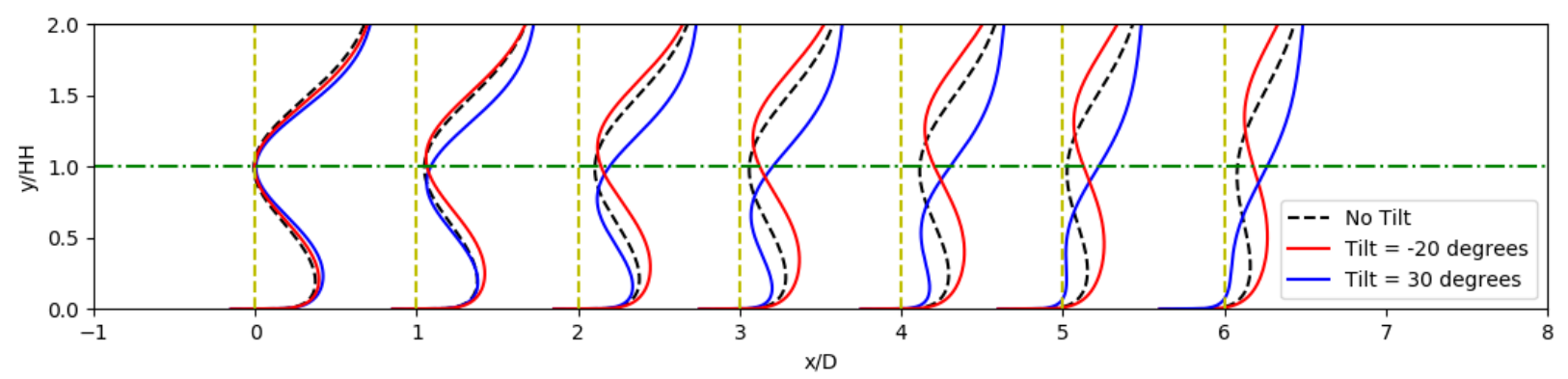

(a) Modified Bastankhah Model

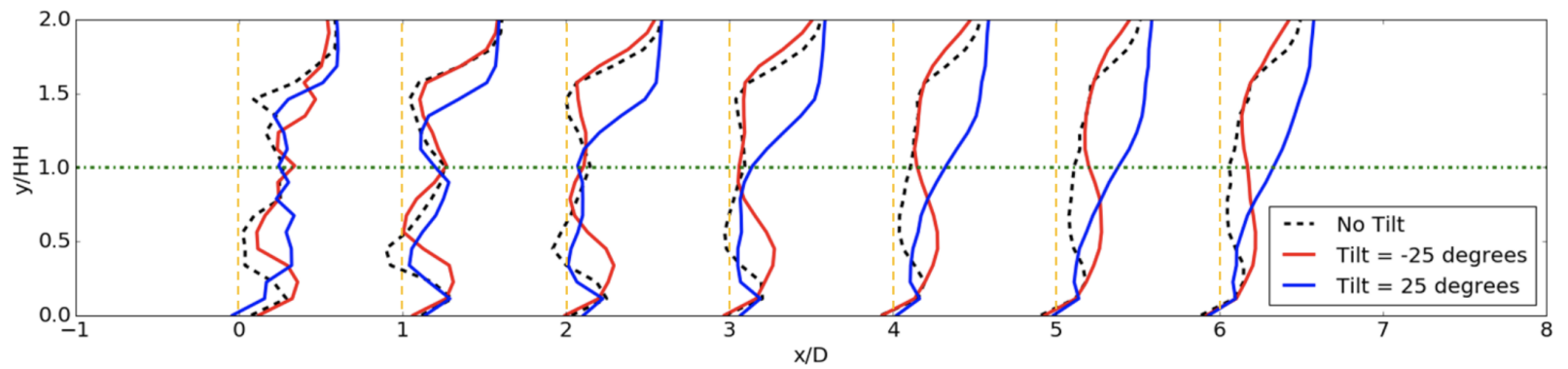

(b) SOWFA

Figure 3 Stream-wise velocity profiles for the modified Bastankhah wake model(a.) and SOWFA(b) [13].

In future work we plan to use more SOWFA runs to further validate our wake model and use physical relationships to account for these adjustments; however, for the optimization scenarios in this paper the performance of the wake model sufficiently matches NREL's SOWFA simulations.

\section{B. Wind Farm Power}

In this section we discuss how we calculate the wind farm power. The power produced by a single turbine is expressed in Eq.7

$$
P_{i}= \begin{cases}0 & V_{\text {eff }}<V_{\text {cut-in }} \\ \frac{1}{2} \rho A_{\text {eff }}\left(V_{\text {eff }}\right)^{3} C_{P} & V_{\text {cut-in }} \leq V_{\text {eff }}<V_{\text {rated }} \\ P_{\text {rated }} & V_{\text {rated }} \leq V_{\text {eff }}<V_{\text {cut-out }} \\ 0 & V_{\text {eff }} \geq V_{\text {cut-out }}\end{cases}
$$

Where $\rho=1.225 \mathrm{~kg} / \mathrm{m}^{3}$ and represents the density of air, $A$ is the rotor swept area (our results used a rotor diameter of 126.4 meters), $C_{P}$ is the turbine power coefficient and correlated to wind speed through NREL's 5MW reference turbine 
$C_{p}$ curve, $V_{\text {cut-in }}=3.0 \mathrm{~m} / \mathrm{s}$ and is the cut-in wind speed, $V_{\text {cut-out }}=25 \mathrm{~m} / \mathrm{s}$ and is the cut-out wind speed, $V_{\text {rated }}=11.4 \mathrm{~m} / \mathrm{s}$ and is the rated wind speed, $P_{\text {rated }}=5.3 \mathrm{MW}$ and is the rated power, and $V_{\text {eff }}$ is the effective wind speed across the rotor hub [17].

The effective velocity across the rotor hub, $V_{\text {eff }}$, is calculated as an average of velocities at 100 points dispersed within the rotor swept area of a turbine. The velocity measured at each one of the 100 points is calculated by taking the free-stream velocity at the height of the point of interest minus the velocity deficit in Eq. 1. The free stream velocity is calculated as a function of height using an exponential wind power curve with a shear exponent of 0.15 .

When a turbine is tilted it will produce less power due to the reduction of the projected rotor swept area. To account for this reduction, the power of each turbine is calculated by multiplying the rotor swept area by $\cos (\gamma)$ to the power of 1.88. The 1.88 value comes from the exponent used in a control study of yaw for NREL's 5-MW turbine operating in SOWFA [10].

$$
A_{\text {eff }}=A \cos (\gamma)^{1.88}
$$

Depending on the direction that a turbine is tilted, the wakes of upstream turbines can be deflected either towards the top or bottom of the downstream turbines. Lower wind speeds at lower heights suggest that the bottom of the rotor swept area is more beneficial to deflect the upstream wakes into. Leaving the upper portions of the rotor swept area exposed to the free stream velocities therefore allowing the turbine to experience a higher average wind speed and consequently produce more power. Deflecting the wake towards the bottom of downstream turbines becomes more beneficial as newer models of wind turbines are being manufactured with larger rotor swept areas.

To validate our wake model we compared the power gains of our wake model with Annoni et al.'s SOWFA simulations for two turbines spaced 7 rotor diameters apart with differing tilt angles in the upstream turbine and variable span-wise positions for the downstream turbine. Figure 4 shows that at 7 rotor diameters downstream our wake model under predicts the maximum power gains for a $25^{\circ}$ tilt in the upstream turbine when the downstream turbine is directly behind it and over predicts power gains for a 1D and -1D span-wise offset of the downstream turbine. This is due in part to the kidney bean shape of the wake in SOWFA with the wake being pushed to the border of the downstream rotor swept area. The modified Bastankhah wake model fairly accurately predicts the decrease in power from tilting the upstream turbine $-25^{\circ}$. This validation shows that our model sufficiently predicts the power gains for our optimization of a wind farm. The under prediction of the max power gain means that the gain in AEP may be under predicted in our optimization and a more accurate model could show more promising power gains.

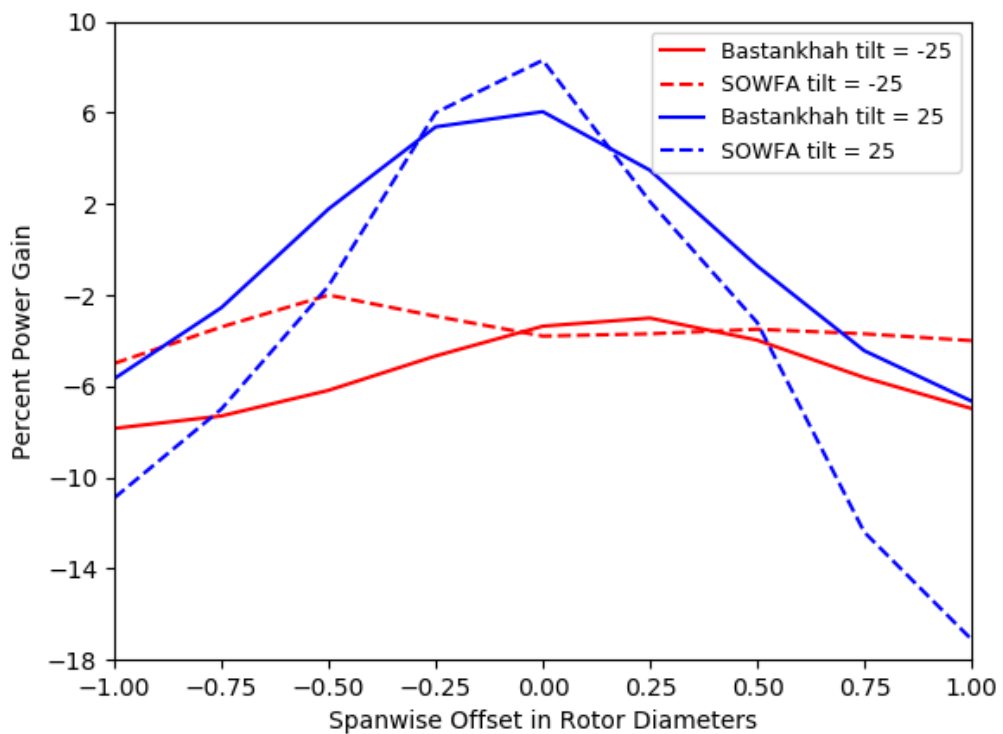

Figure 4 Percent power gain of a two-turbine array with the upstream turbine tilted $-25^{\circ}$ or $25^{\circ}$ compared to the upstream turbine tilted $-5^{\circ}$. These power gains are compared over different span-wise offsets of the downstream turbine. 
To further demonstrate the potential power gains in tilt control we used the same two turbine array as in Fig. 4 except we kept the downstream turbine directly behind the upstream turbine and adjusted the tilt angle of the upstream turbine. This models the benefit of tilting a wind turbine in the positive direction rather than the negative direction. Fig. 5 shows that for tilt angles around $20^{\circ}$ to $25^{\circ}$ the model predicts about a $6 \%$ increase in the total power whereas with a negative tilt angle around $-20^{\circ}$ to $-30^{\circ}$ the model predicts a decrease in the total power by about $-6 \%$.

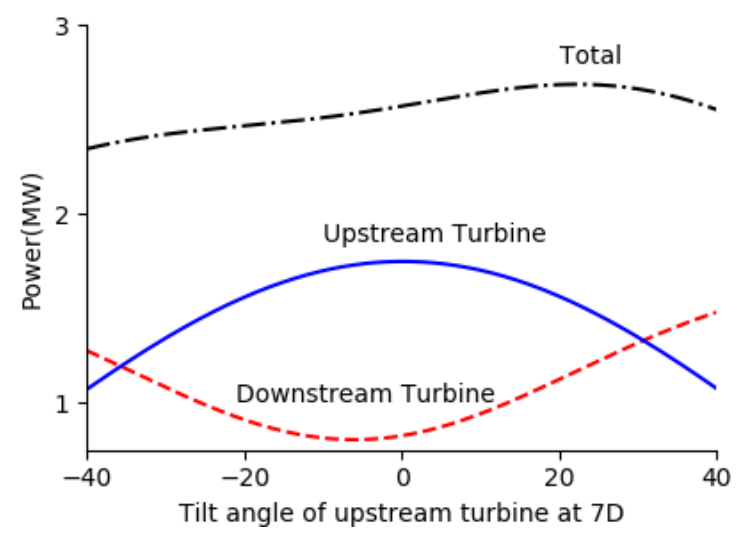

Figure 5 Generated power of a two turbine array spaced 7 rotor diameters apart for differing tilt angles in the upstream turbine.

\section{Optimization}

In order to find the optimal tilt angles for maximum AEP of a wind farm, we used a sparse non-linear optimization algorithm called SNOPT [18]. SNOPT uses a sequential quadratic programming (SQP) algorithm where search directions, effectively solved for using quadratic programming subproblems, minimize the number of evaluations needed to converge to a solution. This is ideal for our optimizations, because there are many variables to control. For the optimizations defined in Eq.9-11 we used 50 random starting points to thoroughly search the function space. The design variables for these optimizations include the tilt $(\gamma)$ and yaw $(\beta)$ angle of each turbine. If the tilt or yaw angle of a turbine isn't being controlled then they are set to default values of $-5^{\circ}$ and $0^{\circ}$ respectively. For active tilt control and active yaw control, the tilt and yaw angle for each turbine is different depending on the inflow direction. For the fixed tilt angle, the individual tilt angles of each turbine are the same for every wind direction. The location, hub height, and rotor diameter for each turbine were fixed. Our optimizations are defined in Eq. 9 - 11 .

$$
\begin{aligned}
& \text { maximize AEP } \\
& \text { w.r.t. } \quad \gamma_{i}(i=1, \ldots, \text { nTurbines) } \quad \text { (fixed tilt angle) } \\
& \text { subject to }-30^{\circ} \leq \gamma_{i} \leq 30^{\circ} \\
& \text { maximize AEP } \\
& \text { w.r.t. } \quad \gamma_{i, j}(i=1, \ldots, \text { nTurbines, } j=1, \ldots, \text { nDirections) } \quad \text { (active tilt control) } \\
& \text { subject to }-30^{\circ} \leq \gamma_{i} \leq 30^{\circ} \\
& \text { maximize AEP } \\
& \text { w.r.t. } \quad \beta_{i, j}(i=1, \ldots, \text { nTurbines, } j=1, \ldots, \text { nDirections) (active yaw control) } \\
& \text { subject to }-30^{\circ} \leq \beta_{i} \leq 30^{\circ}
\end{aligned}
$$

The tilt angle and yaw angle were constrained to a maximum of $30^{\circ}$ in the negative and positive direction. A tilt angle of $-30^{\circ}$ may be extreme, however as stated earlier this study is to explore the possible benefits of tilt optimization with the assumption that such a tilt angle could be possible. If this study and future studies show large increases in AEP, especially in comparison to yaw control, it may be worthwhile to design a wind turbine capable of large tilt angles. The wind farm used to run these optimizations is the Princess Amalia wind farm where the wind rose data is detailed in Fig. 6. 

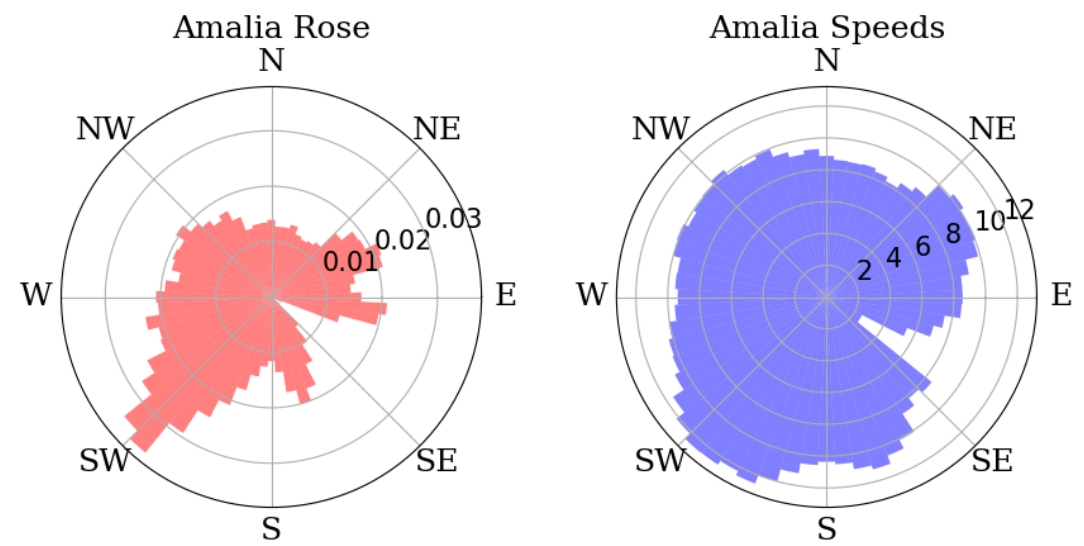

Figure 6 Directionally averaged wind speeds $(\mathrm{m} / \mathrm{s})$ and associated frequencies and directions for the Princess Amalia Wind Farm [19].

\section{Results}

To demonstrate the potential benefits of tilt in a more realistic scenario we optimized the AEP of the Princess Amalia wind farm layout populated with 60 NREL 5 MW reference turbines. The Princess Amalia wind farm was specifically chosen because it receives a fairly consistent amount of high wind speeds from the south-west. This wind farm represents ideal conditions for wake steering with fixed tilt angles due to its potential to have the wind turbines in the south-west deflect their wakes from downstream turbines during the peak wind speeds indicated in Fig. 6 . If the fixed tilt optimization results in significant power gains then the option of fixed tilt angles could also result in power gains for similar wind farms with similar wind resource. The details of the turbine parameters can be found in section B of the proposed methodology section.

\section{A. Fixed Tilt}

Optimizing the AEP of this wind farm with respect to the fixed tilt angle of each turbine resulted in an increase in AEP of $2.77 \%$ (Table 1). The optimized AEP was compared to the AEP of a wind farm under the same conditions with a default of $-5^{\circ}$ tilt in all of the turbines.

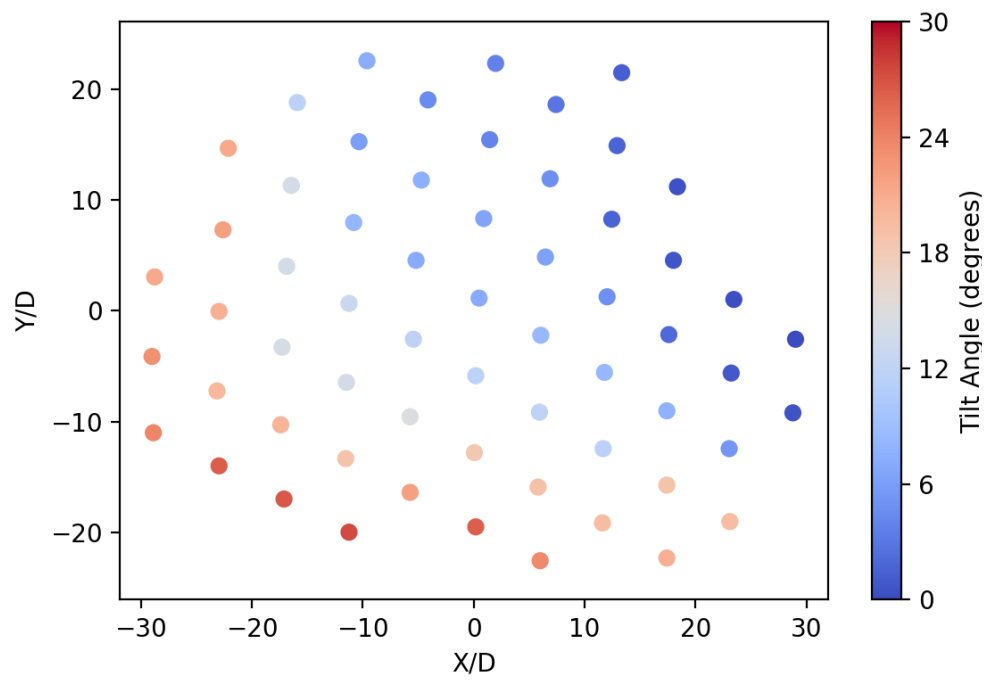

Figure 7 Tilt angles of each turbine for optimizing the AEP of the Amalia wind farm adjusted so that the turbines are about 6D from each other. 
The AEP increases as more upstream turbines are tilted. Figure 7 shows that the south-west side of the wind farm has larger tilt angles than the north-east side. There are larger tilt angles on the south-west side because the wind rose in Fig. 6 shows that higher wind speeds from the south-west occur more frequently than the lower wind speeds from the north-east. The constraints for the tilt angles allowed for negative angles down to $-30^{\circ}$, however, the optimization resulted in only positive tilt angles. This agrees with the same pattern of tilt needed to increase power production in Fig. 5.

In Fig. 7 , there is a row of wind turbines along the south-west border that have fixed tilt angles from around $18^{\circ}$ to $30^{\circ}$. Rather than having a linear change in tilt angles from wind turbines in the south-west to the north-east, this pattern of large tilt angles along the south-west border suggest that large positive tilt angles on the wind dominant side increases power enough that even when the wind isn't coming from the south-west the power gained is more than the power lost. A linear change in tilt angles from the south-west to the north-east may not increase power production enough for the power lost in reduced rotor swept areas due to tilt. In terms of manufacturing fixed tilt angles, this would suggest that there may only need to be turbines with extreme tilt angles along the wind dominant side of a wind farm in order to increase the AEP.

\section{B. Active Yaw}

Optimization of the AEP using active yaw control resulted in a 7.75\% increase in the AEP. This is significantly more than the $2.77 \%$ increase in AEP using fixed tilt angles. In this case and other similar wind farms it may be more cost-effective to use active yaw control considering most wind turbines have the capability to actively yaw and it would be difficult to manufacture the extreme tilt angles in Fig. 7. However, if active tilt control proves to increase the AEP significantly more than active yaw control then it may be beneficial to develop the capability to actively control tilt.

\section{Active Tilt}

Optimization of the AEP of the wind farm using active tilt control increased the AEP by 13.64\% (Table 1). This is a significant increase in AEP compared to the $2.77 \%$ increase in using fixed tilt positions. More importantly, the power gain from active tilt control was significantly higher than the power gain from active yaw control. This large difference in power gain could be attributed to the fact that active yaw control simply redirects wakes in the span-wise direction, however, active tilt control can redirect the wakes towards the ground. Yawing a wind turbine may deflect the wake from a downstream turbine, but it could also push the wake into another turbine. Tilting a wind turbine can deflect the wake towards the ground and away from all downstream turbines.

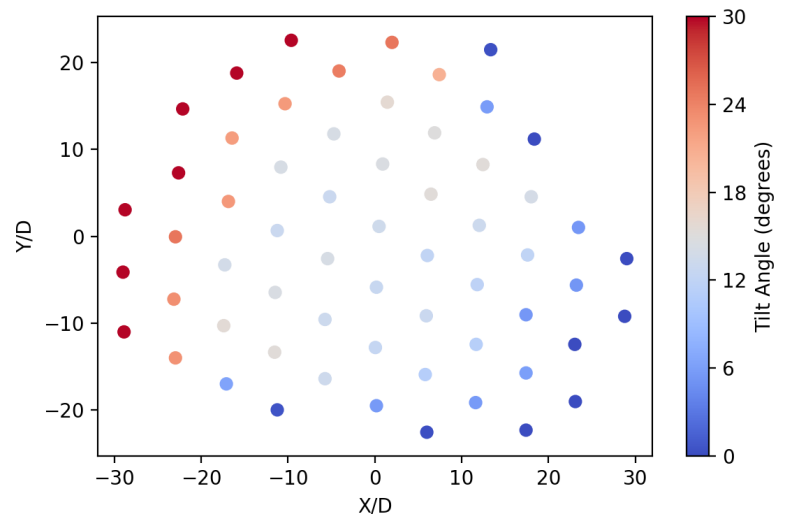

(a) North-west

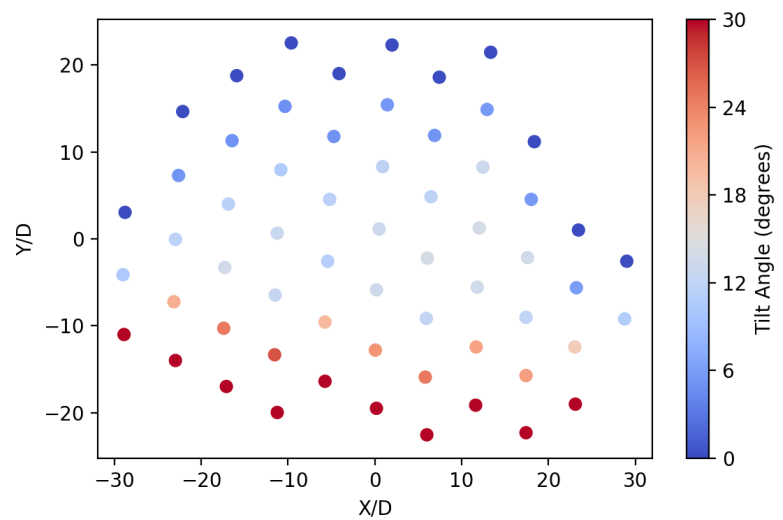

(b) South

Figure 8 Active tilt angles of each turbine for optimizing the AEP of the Amalia wind when the wind is coming from the (a) North-West and the (b) South.

Another possible contributor to the large difference in power gains between active yaw and tilt is the effect of the ground on the wake. The modified Bastkanhah wake model used in the optimizations doesn't include a ground effect, but in calibrating the model to match NREL's SOWFA runs we included a tilt angle offset of $5^{\circ}$. This offset may account 
Table 1 Percent gain in the AEP based on optimization with fixed tilt angles, active tilt control, and active yaw control.

\begin{tabular}{lc}
\hline Method & Percent Gain \\
\hline Fixed Tilt & 2.77 \\
Active Tilt Control & 13.64 \\
Active Yaw Control & 7.75 \\
\hline
\end{tabular}

for some of the ground effect in the optimization scenarios used in this paper, but may not accurately model the ground effect for different scenarios. In order for this model to be more applicable to other wind turbines and wind farms it would need to be recalibrated, however development of a more analytical tilt model that more accurately models the effect of the ground on the wake would be ideal. Such efforts are underway but still need more development to reach an analytical model that can be used in optimizations [20].

It is interesting to note the differences in tilt configurations when comparing the optimization of the tilt angles for one wind direction, as in Figure 8, to the optimization of fixed tilt angles for all wind directions, as in Figure 7 When using active tilt control the pattern for optimal tilt angles shows a barrier of positive tilt angles in the upstream turbines similar to the barrier formed with fixed tilt angles, except the upstream active tilt angles are greater than the upstream fixed tilt angles by about $3^{\circ}$ to $5^{\circ}$. They both also show a similar decrease in tilt angles to $0^{\circ}$ in more downstream turbines, except that the active tilt angles decrease to about $0^{\circ}$ close to the most downstream turbines, whereas the fixed tilt angles decrease to about $0^{\circ}$ closer to the middle of the wind farm.

\section{Conclusion}

In order to assess the potential benefits of tilt control capabilities in a wind farm we optimized the Princess Amalia wind farm with respect to fixed tilt angles, active tilt control, and active yaw control. The Bastankhah wake model used in the optimization was calibrated to match the wake shape for the tilt angles used in Annoni et al.'s SOWFA runs [13]. Annoni et al. compared the power gains from changing tilt in the upstream turbine of a two-turbine array spaced 7 rotor diameters apart. Under the same scenario, the modified Bastankhah model indicated that a tilt angle in the upstream turbine around $20^{\circ}$ to $25^{\circ}$ can increase the total power production of a two-turbine array in line with the wind direction by about $6 \%$ compared to having no tilt in the upstream turbine. However, a tilt angle of about $-25^{\circ}$ can result in a $6 \%$ decrease in power. These observations with the modified Bastankhah model match the patterns of power gain seen in Anonni et al.'s use of SOWFA to estimate the power gains for the same scenarios(see Fig. 55).

Using the modified Bastankhah model that was calibrated with SOWFA, the optimizations with fixed tilt and active tilt control further revealed that tilt capabilities in wind turbines can be advantageous to increasing the AEP of a wind farm even when considering an entire wind distribution. Optimization of the AEP of the Princess Amalia wind farm resulted in a 2.77\% increase in AEP with fixed tilt angles, $7.75 \%$ increase in AEP with active yaw control, and a $13.64 \%$ increase in AEP with active tilt control. The power gain of $2.77 \%$ with fixed tilt angles suggest manufacturing turbines with a fixed tilt angle may not be as beneficial as using other fixed methods such as variable tower heights where manufacturing would be much more simple [5]. However, in comparing active tilt control and yaw control there is a great benefit to active tilt control. The modified Bastankhah model may even under predict the potential power gains of tilt due to not including the effect of the ground on the wake, therefore, a more accurate model could reveal further the benefits of active tilt control. If this large increase in power production doesn't justify building an active tilt mechanism it may be justifiable to build an active tilt mechanism which can be paired with active yaw.

In future work we will further validate our modified Bastankhah wake model by using SOWFA to further match physical phenomena in tilting wind turbines under differing parameters such as veer, wind speed, turbine spacing, and turbulence intensity. We will also look into including ground effects on wake recovery. A major benefit of vertical wake deflection is being able to dissipate turbine wakes faster with ground effects. A turbulence distribution will approximate this phenomenon. The current wake model underestimates the power gains due to wakes being directed into the ground. Therefore when the wake model is more calibrated it should predict a faster wake recovery for such wakes and even larger increases in AEP. We will also consider several more wind farm layouts, turbine spacings, and wind distributions to understand the benefits of tilt optimization in different circumstances. 


\section{References}

[1] Barthelmie, R. J., Frandsen, S. T., Nielsen, M., Pryor, S., Rethore, P.-E., and Jørgensen, H. E., "Modelling and measurements of power losses and turbulence intensity in wind turbine wakes at Middelgrunden offshore wind farm," Wind Energy, Vol. 10, No. 6, 2007, pp. 517-528.

[2] Barthelmie, R. J., Hansen, K., Frandsen, S. T., Rathmann, O., Schepers, J., Schlez, W., Phillips, J., Rados, K., Zervos, A., Politis, E., et al., "Modelling and measuring flow and wind turbine wakes in large wind farms offshore," Wind Energy, Vol. 12, No. 5, 2009, pp. 431-444.

[3] Barthelmie, R. J., and Jensen, L., "Evaluation of wind farm efficiency and wind turbine wakes at the Nysted offshore wind farm,” Wind Energy, Vol. 13, No. 6, 2010, pp. 573-586.

[4] Briggs, K., "Navigating the complexities of wake losses," Norik American Windpower, Vol. 10, 2013.

[5] Stanley, A. P. J., Ning, A., and Dykes, K., "Optimization of Turbine Design in Wind Farms with Multiple Hub Heights, Using Exact Analytic Gradients and Structural Constraints," Wind Energy, Vol. 22, No. 5, 2019, pp. 605-619. doi:10.1002/we.2310.

[6] Gebraad, P. M., Teeuwisse, F., van Wingerden, J.-W., Fleming, P. A., Ruben, S. D., Marden, J. R., and Pao, L. Y., "A data-driven model for wind plant power optimization by yaw control," 2014 American Control Conference, IEEE, 2014, pp. 3128-3134.

[7] Fleming, P. A., Ning, A., Gebraad, P. M., and Dykes, K., "Wind plant system engineering through optimization of layout and yaw control," Wind Energy, Vol. 19, No. 2, 2016, pp. 329-344.

[8] Fleming, P., Annoni, J., Shah, J. J., Wang, L., Ananthan, S., Zhang, Z., Hutchings, K., Wang, P., Chen, W., and Chen, L., "Field test of wake steering at an offshore wind farm," Wind Energy Science, Vol. 2, No. 1, 2017, pp. 229-239.

[9] Gebraad, P., Thomas, J. J., Ning, A., Fleming, P., and Dykes, K., "Maximization of the Annual Energy Production of Wind Power Plants by Optimization of Layout and Yaw-Based Wake Control,” Wind Energy, Vol. 20, No. 1, 2017 , pp. 97-107. doi:10.1002/we.1993.

[10] Gebraad, P., Teeuwisse, F., Van Wingerden, J., Fleming, P. A., Ruben, S., Marden, J., and Pao, L., "Wind plant power optimization through yaw control using a parametric model for wake effects—a CFD simulation study," Wind Energy, Vol. 19, No. 1, 2016, pp. 95-114.

[11] Guntur, S., Troldborg, N., and Gaunaa, M., "Application of engineering models to predict wake deflection due to a tilted wind turbine," EWEA 2012-European Wind Energy Conference \& Exhibition, European Wind Energy Association (EWEA), 2012.

[12] Churchfield, M., Lee, S., and Moriarty, P., "Overview of the simulator for wind farm application (SOWFA)," National Renewable Energy Laboratory, 2012.

[13] Annoni, J., Scholbrock, A., Churchfield, M., and Fleming, P., "Evaluating tilt for wind plants," 2017 American Control Conference (ACC), IEEE, 2017, pp. 717-722.

[14] Bastankhah, M., and Porté-Agel, F., "Experimental and theoretical study of wind turbine wakes in yawed conditions," Journal of Fluid Mechanics, Vol. 806, 2016, pp. 506-541.

[15] Abkar, M., Sørensen, J. N., and Porté-Agel, F., "An analytical model for the effect of vertical wind veer on wind turbine wakes," Energies, Vol. 11, No. 7, 2018, p. 1838.

[16] Xie, S., and Archer, C., "Self-similarity and turbulence characteristics of wind turbine wakes via large-eddy simulation," Wind Energy, Vol. 18, No. 10, 2015, pp. 1815-1838.

[17] Niayifar, A., and Porté-Agel, F., "Analytical modeling of wind farms: A new approach for power prediction," Energies, Vol. 9, No. 9, 2016, p. 741.

[18] Gill, P. E., Murray, W., and Saunders, M. A., "SNOPT: An SQP algorithm for large-scale constrained optimization,” SIAM review, Vol. 47, No. 1, 2005, pp. 99-131.

[19] Brand, A., Wagenaar, J., Eecen, P., and Holtslag, M., "Database of measurements on the offshore wind farm Egmond aan Zee," 2012.

[20] Bay, C. J., Annoni, J., Martínez-Tossas, L. A., Pao, L. Y., and Johnson, K. E., "Flow Control Leveraging Downwind Rotors for Improved Wind Power Plant Operation,” 2019 American Control Conference (ACC), IEEE, 2019, pp. $2843-2848$. 